\title{
ENVIRONMENTAL MONITORING SYSTEMS USING WIRELESS SENSOR NETWORKS: AN OVERVIEW
}

\author{
Saja S. Hasan ${ }^{1}$ \\ ${ }^{*}$ Yasin Yousif Mohammed ${ }^{2}$ \\ 1) M.Sc. Student, Electrical Engineering Department, Mustansiriyah University, Baghdad, Iraq. \\ 2) Department of Electrical Engineering, AL- Mustansiryah University, Baghdadi, Iraq
}

\begin{abstract}
Wireless Sensor Networks (WSNs) consist of wirelessly sensor nodes that set with radio interface which they communicated via it with one another to form a network. Sensor nodes enable environment monitoring and data processing. The advance in both wireless sensor and data processing technologies gives environmental management systems the ability to monitor real-time remote location. Developments in sensor's technologies like embedded systems, distributed processing, Micro Electro Mechanical Systems (MEMS), wireless communications, and wireless sensor applications have contributed the transformation in WSNs recently. WSNs have improved work performance and they have been used in many fields especially for survey and monitoring in environmental monitoring systems (EMSs). This paper aims to introduce a brief overview of wireless sensor networks (WSNs) that are used in the applications of environmental monitoring systems (EMSs).
\end{abstract}

Keywords: Wireless Sensor Networks, Sensors, environment.

\section{Introduction}

Low-cost, low-power, small in size, multifunctional, compact sensor nodes have developed due to the advance in wireless communications and electronics. These sensor nodes have the ability to communicate within small regions. WSNs are considered as one of the important technologies that can change the future by connecting the physical and virtual worlds and enable interacting between them [1]. WSNs consist of few tens to thousands sensor nodes that work together for monitoring a region to obtain and collect data. Two types of WSNs are known: structured and unstructured. Unstructured WSNs contain a collection of sensor nodes that deployed in ad-hoc manner in the region, thus sensor nodes can be dropped randomly from a plane into the desired region. In structured WSNs on the other hand, sensor nodes are deployed in a pre-determined points in the region. Structured WSNs have fewer nodes and they need lower network maintenance and management costs [2]. WSNs are important technology that have a great potential to improve many military and civilian applications. WSNs are used in medicine, transportation, home, precision agriculture, industrial process control, wireless data acquisition as well as creating new systems in environmental monitoring like environmental contaminant detections, smart buildings and cities, target tracking, habitat monitoring, and numerous future military applications[3].

\section{Wireless Sensor Network Components}

A typical architecture of WSN is shown in Figure 1. It consists of inexpensive, tiny and generally simple wireless sensor nodes and a base station 
(BS) [4]. There are three types of topologies in sensor networks; Star, Mesh and Tree topology [3]. A base station, which is sometimes referred to as the sink, is the point of data collection in WSN. It acts as a network-layer gateway to another network, a data processing or storage center. Moreover, BS is considered as an access point for human interfaces or it can be used as a connection to send control information into the network or get data from it. A sensor network contain many sensor nodes for each BS. Sometimes there may only be a single BS for the entire sensor network. When the BS receives the collected and sensory data from the wireless nodes, it will forward these data to the task manager node using Internet or satellite communications.

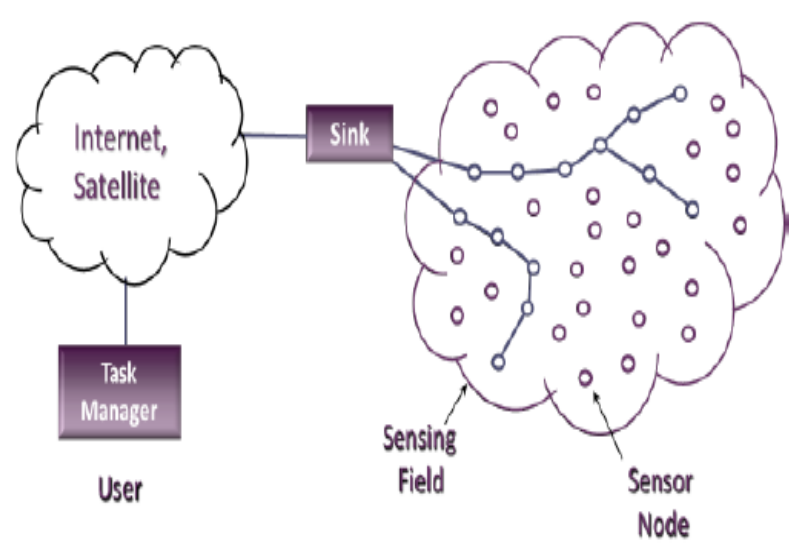

Figure 1. Typical Architecture of Wireless Sensor Network [5]

Wireless sensor nodes are very small devices that have the ability to:

a) Interact with the environment by sensing, monitoring and controlling physical environmental parameters from remote locations.

b) Perform some computational data processing c) Communicate with other neighboring nodes in the network for implementing a specific task.

d) Store the collected data because each sensor node has some storage capacity.

One the most important issues that affect the design of these small, battery-powered sensor nodes is the way in which the power is consumed by these nodes because each one of the nodes spend most of the energy it has in the communication process with the other nodes. To avoid consuming a lot of power, sensor nodes require mechanisms for minimizing their limited energy consumption and ensuring long-lasting and unattended operations in remote regions without the need for replacement or continuously recharging their batteries. Also it is preferred to put the transceiver unit of the sensor node in the sleep mode whenever it's possible [5]. Sensor nodes are deployed inside the observation region either in an ad hoc or in a pre-planned way. Sensor nodes in the deterministic deployments are placed and the collected data is routed across pre-determined paths. Random node deployment on the other hand, involves sensor nodes are scattered and deployed randomly in an ad hoc structure. Each sensor node consists of the following main components:

1-Microcontroller unit: used to perform and implement tasks and local computations on the sensed data, it's responsible on collected data processing and it assists other components of the sensor node in their functions.

2-Transceiver unit: It implements a combination of the transmitter and the receiver functions.

3-Sensing unit: which senses the environment

4-Memory unit: This unit is mainly used for collected data storage

5- Power unit. 
The sensor board consists of large number of sensors that have different types like low sampling rate magnetic sensors, infrared, thermal, visual, acoustic, and radar sensors. Using any type of these sensors depends on the engineering and design of the WSNs for specific fields deployments [5]. Figure 2 shows that different wireless sensors network topologies.
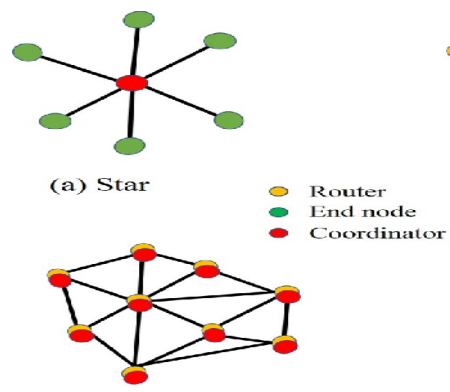

(c) Digimesh

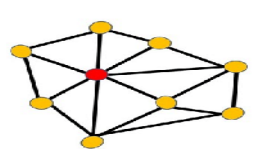

(b) Peer to Peer Mesh

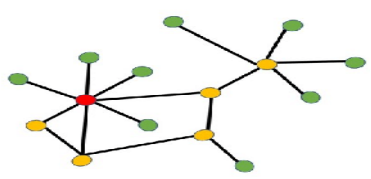

(d) Zigbee
Figure 2. Different WSNs topologies.

\section{Environment Monitoring System}

One of the important WSNs applications is the Environmental monitoring which may contribute large effects. These applications grow widely with this developed technology. Generally environmental monitoring systems (EMSs) are designed and used for sensing, monitoring and controlling environment parameters such as temperature, light, pressure and humidity. The unstable weather conditions make it very important to understand the surrounding environment and its effect on the human being. EMSs include all the processes, actions and methods of data collection that used for observing, sensing and monitoring the changeable environmental conditions [6]. Data that represent natural weather conditions such as temperature, light, and pressure are collected using sensors that deployed over large regions, then these data are transmitted to a server.

\section{Environment Applications}

EMSs has been applied in different and multiple applications that assist people in their daily works and develop their manner of life in the future. Using EMSs help to reduce cost and time. EMSs have grown rapidly in multiple and useful applications such as agricultural monitoring, habitat monitoring, poisonous gases monitoring, indoor monitoring, greenhouse monitoring, radiation monitoring, climate monitoring and forest monitoring. Nowadays, the community begin to realize the importance and the advantages of using and developing the WSN technologies in their daily life [7]. Major WSNs Environmental applications are :

1-Precision agriculture Monitoring: It is an emerging WSNs application aspect that is used for monitoring, sensing and controlling the amount of pesticides, monitoring crop, soil and climate parameters in large agricultural fields[7].

2- Greenhouse Monitoring: This system is important for monitoring solar radiation and ensuring the stabilization of the environmental parameters. It can be a web-based and remote controlled system to allow easily access for the user in order to control, sense and monitor greenhouse using Internet connection. The system gives the opportunity for the user to access and control the data collected from everywhere [8].

3-Climate Monitoring: There are many changes in the climate all over the world nowadays. These changes caused many serious effects like the sea ice breaking, increasing in the level of sea water, lake temperature warming, heat waves, glacier melting and many other phenomena that urged efforts to develop systems helping control and monitor the climate changes [9]. 
4- Forest Monitoring: Forests are important and essential sources for the ecological balance and biodiversity, so it has been very necessary to implement a system for monitoring the environmental parameters in the forest regions. There are several studies that highlighted the subject of forest monitoring system [7].

5- Air Pollution monitoring: It is one of the very important application of EMSs although it has been considered as a very complicated task. Over the past years, data were collected and recorded periodically. This manner consumed very long time and also it was very expensive. Nowadays, using WSNs make air pollution monitoring process more efficient, less complex, and the recording of data readings became more instantaneous and accurate[10] .

6- Flood and Fire Detection: One of the multiple environmental applications that developed using WSNs is detecting and monitoring forest fires by the deployment of sensor networks in the forest in order to detect the origin of such destroying fires [11]. The same idea has been applied for flood detection using weather sensors that are used in the flood detection and monitoring system to detect, sense, predict and as a result prevent floods or reduce its effects [7].

7-Radiation Detection: The establishment of WSNs for radiation monitoring has gained a lot of attention in many countries all over the world. The use of these systems may play an important role in the assistance of security forces and other authorities that concerned with radiation detection to determine and monitor the levels of radiation in the infected regions and areas as well as avoid compromising workers life's [12].

\section{Advantages of Using WSNS}

1. Low cost
2. Minimum power consumption comparing it with traditional wired networks.

3. easy in expanding or reducing the coverage region.

4. No need for wiring.

5. Small size.

6. suitable for recent applications.

7. Centralized and localized monitoring system.

\section{Disadvantages Of Using WSNS}

1. Lower speed comparing it with traditional wired networks

2. less secure and easy to hack by hackers.

3. Affected by long distance and obstacles such as Walls.

\section{Challenges Face WSNS}

The essential requirements that must be taken in consideration when deploying WSNs to get a good, stable and efficient monitoring system are as follows:

1-Power consumption: wireless sensor nodes are the most suitable and popular solution for monitoring applications. However, because these wireless sensor nodes are generally located in a hard and difficult to reach positions, the process of changing the battery regularly will not be easy and it will be inconvenient. In order to avoid this problem, it is very important to make sure that there's always an adequate power accessible for the system [3], also it is essential to make sure that the batteries used in the system are capable to function in proper manner for long time because the radio transceiver unit of the WSNs consumes big amounts of power. 
2-Reliability: WSNs must be simple handling and have predictable operations to avoid sudden and unexpected crashes that may happen. Another matter must be taken in to consideration is avoiding the maintenance of WSNs by persons who don't have experience because end user may have no knowledge on WSNs. Changes on the region of interest may happen while transmitting packets of data. Therefore, achieving reliability is very essential to prevent packets loss[7].

3-Security: This term is used widely. It encompasses authentication, integrity, nonrepudiation, anti-playback, and privacy. As the dependency on the information and data obtained from the WSNs increased, the potential risk of hacking these information by hackers during the transmission over networks has increased. Several techniques such as steganography and cryptographic are implemented to ensure the security for transmission of these data over WSNs [3].

4-Robustness: WSNs must have robustness in order to be able to encounter hardware failure problems and the poor signal connectivity problems. For example, the effects of environmental parameters such as rain, humidity and windstorm may cause short circuit problems and rebooting the system [7].

5-Flexibility: Specialist user must have the ability to make changes in the stations of WSNs such as addition, replacing, or moving stations at any time to satisfy the requirement of WSNs. For example, the user may need to change the location of some stations when the current location of the nodes become out of range to transmit signals.

\section{Conclusion}

Wireless Sensor Networks (WSNs) are networks that consist of multiple deployed sensors which is responsible for sensing and monitoring an event then transmitting the collected data wirelessly to the sink or base station. In this paper some of WSNs applications that appear recently and focus on environment monitoring systems (EMSs), are surveyed. The need for using WSNs is increasing rapidly as a result to the fact that the use of these networks have opened the way to perform many critical tasks that is difficult or even impossible for humans to perform. WSNs have improved the performance and robustness. They also provide high efficient monitoring systems. To conclude, EMSs are expected to have a very important role in the future daily life. EMSs are proved to be an efficient alternative way that will replace the traditional ways that are used depending on men force for monitoring the environmental parameters. However, these systems still need improvements from different aspects such as privacy and security.

\section{Conflict of interest}

The publication of this article cause no conflict of interest.

\section{References}

1. Cao, X., Chen, J., Zhang Y. and Sun,Y, Development of an integrated wireless sensor network micro-environmental monitoring system, ISA Transactions, 2008.

2. Deborah, E., Ramesh,G., John, H. and Satish, K, Next century challenges: scalable coordination in sensor networks, In Proceedings of the 5th annual ACM/IEEE international conference on Mobile computing and networking Seattle, Washington, United States: ACM Press, 1999.

3. M. Logambal, Dr. V. Thiagarasu, Applications Of Wireless Sensor Networks: An Overview, International Journal Of Engineering Sciences \& Research Technology, 2017. 
4. Akyildiz, I. F., Su, W., Sankarasubramaniam, Y., \& Cayirci, E., Wireless Sensor Networks: A Survey, Computer Networks, 2002.

5. Eirini Karapistoli, Ioanna Mampentzidou, and Anastasios A. Economides, Environmental Monitoring based on the Wireless Sensor Networking Technology: A Survey of Real-World Applications, International Journal of Agricultural and Environmental Information Systems, 2014.

6. G. Barrenetxea, F. Ingelrest, G. Schaefer, and M. Vetterli, Wireless Sensor Networks for Environmental Monitoring: The SensorScope Experience, IEEE International Zurich Seminar on Communications, 2008.

7. Mohd Fauzi Othmana, Khairunnisa Shazalib, Wireless Sensor Network Applications: A Study in Environment Monitoring System, International $\mathrm{S}$ ymposium on $\mathrm{R}$ obotics and Intelligent $\mathrm{S}$ ensors, 2012.

8. I. Matijevics, Wireless Sensors Networks Theory and Practice, Towards Intelligent Engineering and Information Technology, 2009.

9. Y. K. Lee, Y. J. Jung, and K. H. Ryu, Design and Implementation of a System for Environmental Monitoring Sensor Network, International workshop, Huang Shan, China, 2007.

10. Kavi K. Khedo1, Rajiv Perseedoss and Avinash Mungur, A Wireless Sensor Network Air Pollution Monitoring System, International Journal of wireless \& mobile Networks (IJWMN), 2010.

11. Y. Liu, Y. Gu, G. Chen, Y. Ji, and J. Li, $A$ Novel Accurate Forest Fire Detection System Using Wireless Sensor Networks, Seventh International Conference on Mobile Ad-hoc and Sensor Networks, 2011.

12. Khairul Anwar Bapujee and Nahrul Khair Alang Md Rashid, Wireless Wide Area
Radiation Monitoring Network Using Internet of Things, mekatronika - Journal of Intelligent Manufacturing \& Mechatronics, 2019. 